v. Graefes Archiv für Ophthalmologie, Bd. 158, S. 1-7 (1956)

Aus dem Neuroophthalmologischen Laboratorium (Dr. J. R. WoLTER) der Abteilung für Neuropathologie (Prof. Dr. K. Scharenserig) der Neuropsychiatrischen Klinik (Prof. Dr. R. W. WAGGONeR) des Universitätskrankenhauses Ann Arbor, Mich., USA

\title{
Zentrifugale (antidrome) Nervenfasern im menschlichen Sehnerven \\ Von
}

\section{J. ReIMER WoLTer und LEOPOLD Liss*}

Mit 5 Textabbildungen

Der Sehnerv gehört strenggenommen nicht zu den Hirnnerven, sondern ist vielmehr sowohl seiner Entwicklung als auch der anatomischen Struktur nach als interzentrale Commissur - also als Teil weißer Hirnsubstanz aufzufassen. Es besteht kein Zweifel daran, daß die Mehrzahl der Nervenfasern, die den Sehnerven aufbauen, zentripetaler Natur sind. Der Funktion nach werden diese zentripetalen (afferenten) Fasern in visuelle Elemente, in solche, die mit der Pupillenreaktion zu tun haben und in den sog. energetischen Anteil der Sehbahn nach HorLwICH (dorsale hypothalamische Opticuswurzel nach FreY) eingeteilt. Keinerlei Einigkeit besteht jedoch in der Literatur über die Frage der Existenz von zentrifugalen (efferenten, antidromen) Nervenfasern im menschlichen Sehnerven.

Zum besseren Verständnis sei hier erwähnt, daß die Ganglienzellen aller zentripetalen Fasern des Sehnerven in der Retina liegen, während die Ganglienzellen der zentrifugalen Fasern im Gehirn oder auch im anderen Auge angenommen werden. Bei allen Fasern des Sehnerven handelt es sich um Neuriten - also um Zellfortsätze, die nur im Zusammenhang mit den zugehörigen Ganglienzellen existenzfähig sind und bei Abtrennung von denselben nekrotisch werden und alsbald spurlos verschwinden.

Die Existenz zentrifugaler Nervenfasern im Sehnerven wird in den modernen Lehr- und Handbüchern der Ophthalmologie and Anatomie teilweise als fraglich hingestellt (z. B. Lauber, Polyak, Friedenwald, KAPPERS, Huber und Grosby), teilweise aber auch als tatsächlich vorhanden angesehen (z. B. GrefF, Cone und Mcmillan, Duke Elder). Dabei begründen GRefF sowie CONE und. MacmILlan ihre positive Einstellung zu der vorliegenden Frage mit den Befunden von CaJal, Tello. Perlia und Wallenberg, die bei Vögeln und beim Kaninchen die

* Mit der Unterstützung des U.S. Departments of Public Health, Education and Welfare, Grant No. B. - 475 (C 2). 
Existenz zentrifugaler Fasern im Sehnerven beschrieben haben. Auch v. MonaKow hat sich für die Existenz zentrifugaler Fasern ausgesprochen. Andererseits betonten WIENER und MüNzER sowie später auch Marburg, daß im Sehnerven des Kaninchens und des Menschen aus-

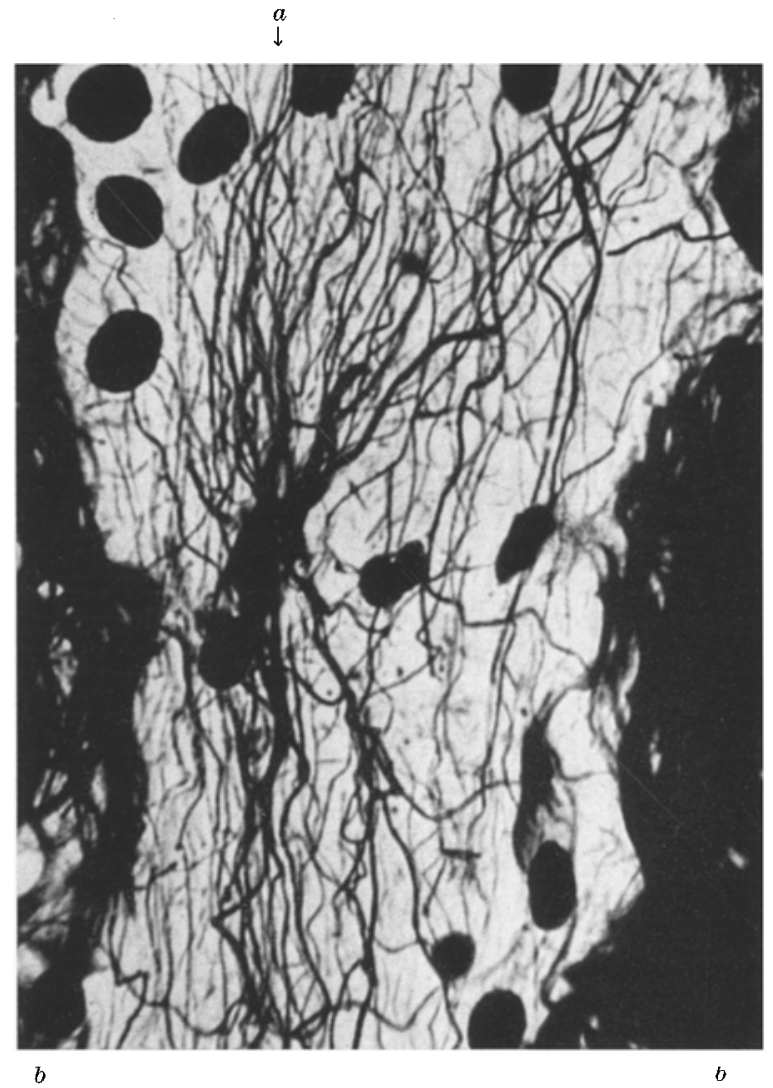

Abb. 1. Längsschnitt des Sehnerven von Ch. S. Hypertrophischer Astrocyt entsendet ein dichtes Gewirr von Gliafasern $(a)$. Bindegewebe der Septen ist tiefschwarz imprägniert $(b)$. Hortega-Methode, Mikrophotographie, Vergr. 900mal

schließlich zentripetale und keine zentrifugalen Nervenfasern vorhanden seien.

Die vorliegenden Befunde von zwei klinischen Fällen scheinen uns bedeutungsvoll als endgültiger Beweis für die Existenz von zentrifugalen Fasern im menschlichen Sehnerven.

\section{Mothodik}

Die Präparate, die der vorliegenden Arbeit zugrunde liegen, wurden von zwei Sehnerven von zwei Patienten hergestellt, die sofort nach der Entnahme bei den Sektionen in Bromformalin fixiert und auf dem Gefriermikrotom in Längsrichtung 
geschnitten wurden. Zur Darstellung der Nervenfasərn und der Glia wurden die Silbercarbonatmethoden DeL Rio HorTegas benutzt, wie sie ScharenBerg in der deutschen Literatur zum Teil verbessert im Zusammenhang beschrieben hat.

Fall 1. Bei dem Patienten Ch. S. wurde an beiden Augen, die wegen purulenter Panophthalmitis nach Hornhautperforation bei malignem Exophthalmus erblindet waren, 1944 durch Herrn Prof. Dr. FraLick ${ }^{1}$ eine Exenteratio oculi vorgenommen. Der Patient starb 1955 - also 11 Jahre nach der Operation. Der rechte Sehnerv

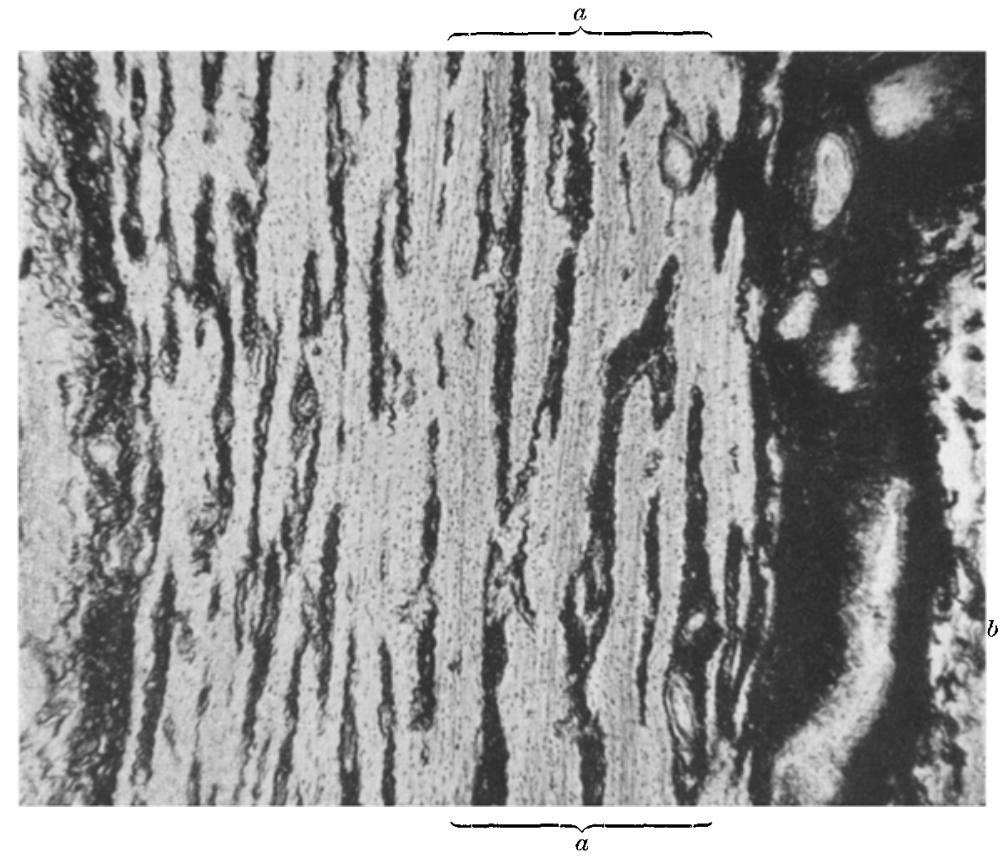

Abb. 2. Längsschnitt aus dem Sehnerven von Ch. S. Der Sehnervenbereich, der noch Nervenfasern enthält, ist deutlich erkennbar $(a)$. An beiden Seiten des Bildes die Piascheide erkennbar, die rechts die in den Sehnerven eintretenden Zentralgefäße enthält (b). HortegaMethode, Mikrophotographie, Vergr. 100mal

wurde nach dem Tode entnommen und uns zur histologischen Untersuchung zur Verfügung gestellt.

Die makroskopische Untersuchung dieses Sehnerven zeigte bereits deutlich eine starke Atrophie. Der Nerv war wesentlich dünner als normal, fühlte sich sehr derb an und lag nur lose in der viel zu weiten Durascheide.

Während normalerweise bei der mikroskopischen Untersuchung die dichten Nervenbündel weitaus die Hauptmasse des Sehnerven aus. machen, stand in diesem Fall hypertrophische Astroglia und Bindegewebe im Vordergrund des histologischen Bildes. Dabei formte die Astroglia eine dichte Narbe von großen, weitverzweigten Astrocyten

1 Direktor der Universitäts-Augenklinik Ann Arbor, Mich. 
zwischen den ebenfalls hypertrophierten bindegewebigen Septen (Abb. 1). Bei der Nervenfaserfärbung zeigte sich überraschenderweise, daß in diesem atrophischen Sehnervenstumpf noch eine beachtliche Anzahl von Nervenfasern erhalten war. Diese Fasern fanden sich besonders in den zentralen Bereichen des Sehnerven, sie waren in spärlichen Gruppen un-

$a$

$\alpha$

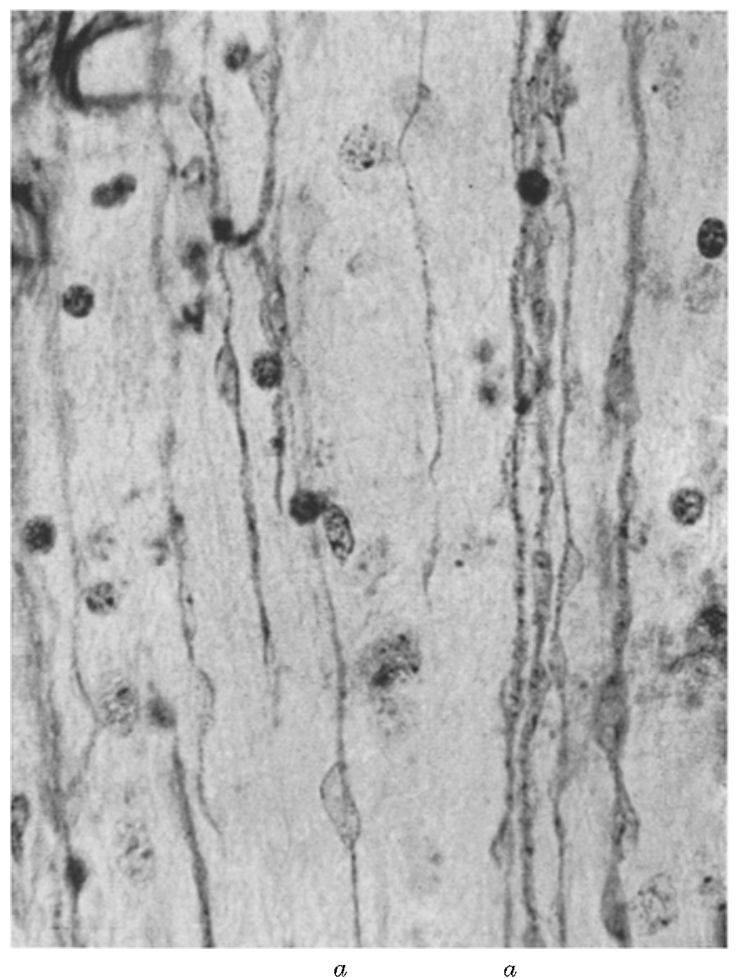

Abb. 3. Längsschnitt aus dem. Sehnerven von Ch. S. Einzelne, feine Nervenfasern bei zarter Versilberung $(\alpha)$. Die Achsencylinder sind nur angedeutet, die Markscheiden mit den perlschnurartigen Schwellungen deutlich zu erkennen. Hortega-Methode, Mikrophotographie, Vergr. 900 mal

regelmäßig angeordnet und verliefen in Längsrichtung des Sehnerven (Abb. 2). Die einzelnen Fasern waren sehr fein (etwa $2 \mu$ dick) und wiesen immer eigentümliche, perlschnurartige Schwellungen ihrer Markscheiden auf (Abb. 3 und 4). Die Nerven waren so zart, daß es sehr ausgiebiger Imprägnation in Silbernitrat bedurfte, um sie darzustellen.

Fall 2. R. S. verlor das rechte Auge 1941 durch Enukleation nach perforierender Verletzung bei einer Bergwerksexplosion. Der Patient starb 1955 im Alter von 70 Jahren - also 16 Jahre nach dem Verlust des Auges. Auch sein rechter Sehnerv konnte nach dem Tode entnommen und histologisch untersucht werden.

Der rechte Sehnerv von R. S. zeigte ebenfalls schon makroskopisch deutliche Atrophie. Der mikroskopische Befund deckte sich im wesent- 
lichen mit dem oben beschriebenen von Ch. S. Auch hier bestand eine dichte, narbige Astroglia zwischen vergröberten Bindegewebssepten. Und wiederum waren feine Nervenfasern erhalten, die in Längsrichtung des Sehnerven verliefen (Abb. 5). Bei entsprechender Färbung zeigten diese Fasern ebenfalls jene perlschnurartigen Anschwellungen der Markscheiden.

$a$

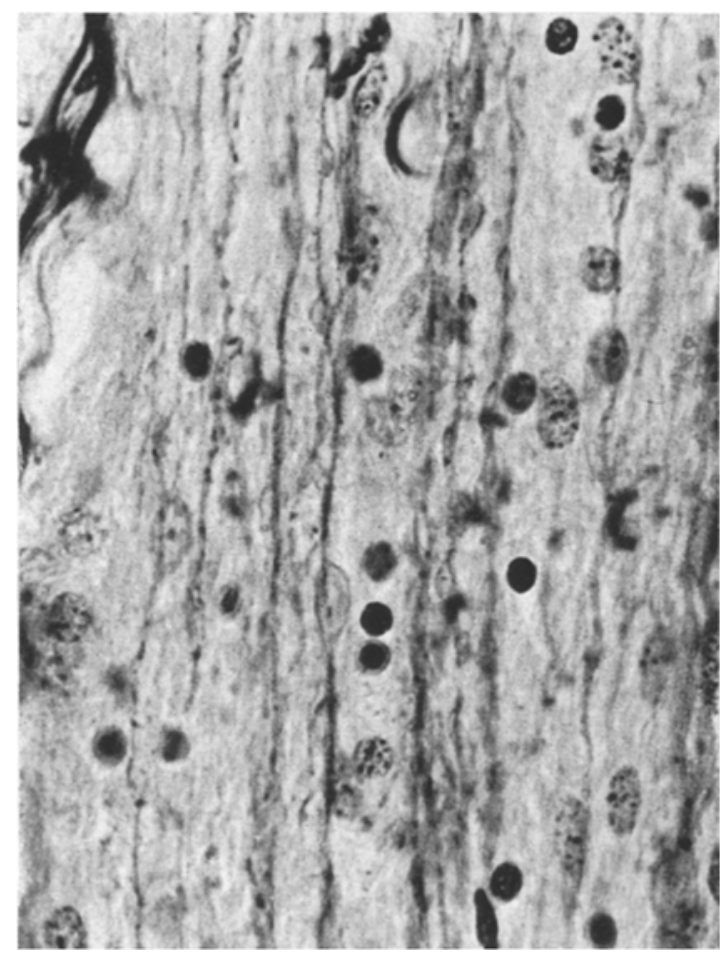

$a$

Abb. 4. Längsschnitt aus dem Sehnerven von Ch. S. Eine Gruppe derselben Nervenfasern wie in Abb. $3(a)$. Hortega-Methode, Mikrophotographie, Vergr. $900 \mathrm{maI}$

\section{Erörterung}

Es handelte sich also um zwei Sehnerven von verschiedenen Patienten, deren zugehörige Retina eimal 11 und einmal 16 Jahre vor der histologischen Untersuchung operativ entfernt wurden. Die Tatsache, daß in beiden Sehnerven noch eine beachtliche Anzahl von Nervenfasern erhalten war, möchten wir als Schwerpunkt dieser Arbeit hervorheben.

In beiden Fällen sind ohne Zweifel mit der Retina die Ganglienzellen aller zentripetalen Nervenfasern des Sehnerven entfernt worden. Die zentripetalen Fasern als Neuriten dieser Zellen müssen also bald nach der Operation degeneriert und zur Zeit der histologisehen Untersuchung längst spurlos verschwunden gewesen sein. Der vorliegende Befund, daß noch 
Nervenfasern in diesen Sehnerven vorhanden waren, kann nur durch die Annahme erklärt werden, daß die Ganglienzellen derselben nicht in den entfernten Retinae gelegen haben. Es muß sich bei den erhaltenen Elementen also um antidrome Fasern gehandelt haben. Als Ursprung solcher Fasern kommen der Literatur nach nur das Gehirn oder die Netzhaut des

$a$

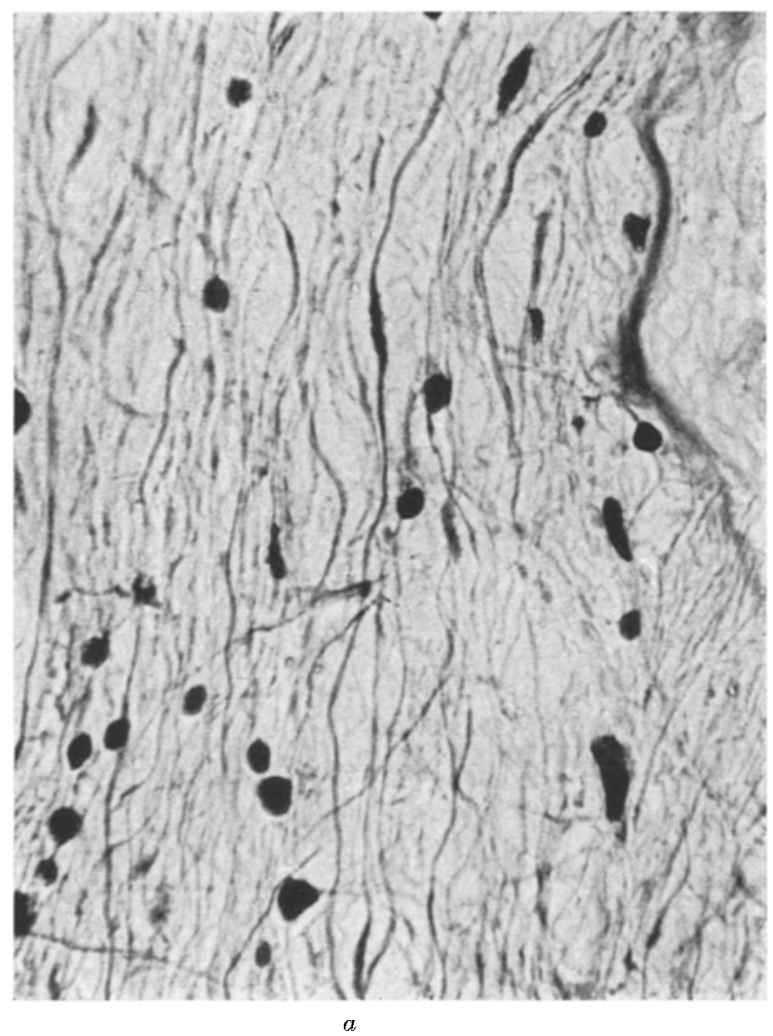

Abb. 5. Längsschnitt durch den Sehnerven von R. S. Finzelne feine Nervenfasern bei gröberer Versilberung der Achsenzylinder $(a)$. Die Markscheiden sind hier nicht dargestellt. $b$ : Bindegewebe. Hortega-Methode, Mikrophotographie, Vergr. 900mal

anderen Auges in Frage. Da in unserem ersten Falle beide Netzhäute entfernt wurden - die Zahl und Art der erhaltenen Nervenfasern aber in beiden Fällen etwa gleich waren - können wir aussagen, daß als Ursprung der hier geschilderten Nervenfasern nur das Gehirn in Frage kommt. Wir sehen den geschilderten Befund als einen Beweis an für die Existenz zentrifugaler, efferenter Fasern im Sehnerven des Menschen.

Während die Dicke der Nervenfasern im normalen menschlichen Sehnerven mit zwischen 2 und $10 \mu$ angegeben wird (LAUBER), fanden wir in unserem Falle Fasern, die etwa $2 \mu$ dick waren. Aus dieser Tatsache wollen wir nicht folgern, daß die zentrifugalen Fasern auch normaler- 
weise so dünn sind. Als Folge der langen Jahre ohne Erfolgsorgan und Funktion mag sehr wohl eine Atrophie der geschilderten Fasern vorliegen. Ebenfalls möchten wir hier nicht weiter auf den Befund der perlschnurartigen Markscheidenschwellungen eingehen. Solche Schwellungen sind auch an vielen Fasern des normalen menschlichen Sehnerven ein regelmäßiger Befund, den wir bisher jedoch nicht ausreichend erklären können. Vom Studium hochgradig phthisischer Augen mit vollständig abgelöster, geschrumpfter und schwer degenerierter Netzhaut wissen wir, daß dieselben Nervenfasern, die wir in den Sehnervenstümpfen der beiden vorliegenden Fälle beobachten konnten, in die Nervenfaserschicht der Netzhaut einstrahlen (unveröffentlicht). Über den weiteren Verlauf, über die Endigungsweise, über die Herkunft im Gehirn und über die Funktion der besprochenen Fasern können wir bisher nichts aussagen. Tierversuche, mit denen wir das Zahlenverhältnis zwischen zentrifugalen und zentripetalen Fasern im Sehnerven festlegen möchten, sind im Gange.

\section{Zusammenfassung}

Sehnerven von zwei Patienten wurden histologisch untersucht, deren zugehörige Retinae einmal 11 und einmal 16 Jahre zuvor operativ entfernt wurden. In beiden Sehnerven wurden gleicherweise noch zahlreiche, feine Nervenfasern erhalten gefunden. Diese Tatsache wird als Beweis für die Existenz zentrifugaler Nervenfasern im Sehnerven des Menschen angesehen.

\section{Literatur}

CAJAL, S., R. y: Degeneration and Regeneration of the Nervous System, Bd. II, S. 584. London: Oxford University Press 1928. - Cone, W., and J. A. MCMULuAN: The optic nerve and papilla. In WILder Penfreud, Cytology and Cellular Pathology of the nervous system, Bd. II, S. 890. New York: P. B. Hoeber Ine. 1932. Duke Elder, Sir St. W.: Text Book of Ophthalmology, Bd. I, S. 102. St. Louis: C. V. Mosby Company 1940. - Friedenwald, J. S., H. C. Wilder, A. E. MadMenee u. a.: Ophthalmic Pathology. An Atlas and Text Book, S. 12. Philadelpia u. London: W. B. Saunders Comp. 1952. - GREFF, R.: Die mikroskopische Anatomie des Sehnerven und der Netzhaut. In GRaEFE-Safmisch, Handbuch der gesamten Augenheilkunde, Bd. I, Abt. II. - KapPers, C. U., C. C. HubER and E. C. CRosBy: The Comparative Anatomie of the nervous system of vertebrates including man, Bd. II, S. 60. New York: The Macmillan Company 1936. - LaUber, H.: Der Sehnerv. In MöLLENDORfFs Handbuch der mikroskopischen Anatomie des Menschen, S. 493 Berlin: Springer 1936. - Marburg, D.: Arch. Ophthalm. 28, 61 (1942). - Monakow, V.: Arch. f. Psychiatr. 14, 699 (1883). - Perlia, W.: Arch. f. Ophthalm. 35, 20 (1889). - Polyak, St.: The retina, S. 340. Chicago: University of Chicago Press 1941. - Scharengerg K., u. W. Zeman: Arch. f. Psychiatr. 188, 430 (1952). - Tello, F.: Trab. Labor. Invest. biol. Univ. Madrid ๖, 237 (1907). - Wallenberg, A.: Neur. Zbl. 17, 532 (1898). - Wiener, E., u. H. MÜNZER: Mschr. Psychiatr. 12, 241 (1902).

Dr. J. Reimer Wolter, Neuropsychiatric Institute, University of Michigan Hospital Ann Arbor, Mich., USA 